\title{
DISTANT FUTURE AND ANALYTIC MEASURES
}

\author{
JUN-ICHI TANAKA ${ }^{1}$ \\ Dedicated to Professor Junzo Wada on his 60th birthday
}

\begin{abstract}
Using a representation of analytic measures in terms of a flow built under a function, it is shown that a positive measure is the total variation measure of an analytic measure if and only if the distant future is the zero subspace. This settles a problem posed by Forelli in connection with his generalization of $\mathrm{F}$. and $\mathrm{M}$. Riesz theorems. We also provide another version of Helson's existence theorem.
\end{abstract}

1. Introduction. Let $X$ be a locally compact Hausdorff space on which the real line $R$ acts as a topological transformation group. This means that there is a one-parameter group $\left\{T_{t}\right\}_{t \in R}$ of homeomorphisms of $X$ with the property that the mapping of $(x, t), T_{t}(x)$, is continuous on $X \times R$. Let $C_{0}(X)$ denote the space of all continuous complex-valued functions which vanish at infinity. The dual space of $C_{0}(X)$ is the space of all bounded complex Baire measures on $X$ and is denoted by $M(X)$. Using $\left\{T_{t}\right\}_{t \in R}$, one may convolve a bounded complex (Baire) function $\phi$ on $X$ or a measure $\lambda$ in $M(X)$ with a function $f$ in $L^{1}(d t)$, the usual Lebesgue space on $R$. The convolution $\phi * f$ is defined by the equation

$$
\phi * f(x)=\int_{-\infty}^{\infty} \phi\left(T_{-t} x\right) f(t) d t
$$

and the convolution $\lambda * f$ is defined to be the measure in $M(X)$ such that, for all $\psi$ in $C_{0}(X)$,

$$
\int_{X} \psi(x) d(\lambda * f)(x)=\int_{X} \psi * \tilde{f}(x) d \lambda(x)
$$

where $\tilde{f}(t)=f(-t)$. The spectrum of a bounded function $\phi$ (resp., a measure $\lambda$ in $M(X))$, in the sense of spectral synthesis, is then defined to be the hull of its annihilator and will be denoted by $\operatorname{sp}(\phi)$ (resp., $\operatorname{sp}(\lambda)$ ). A bounded function $\phi$ (resp., a measure $\lambda$ in $M(X)$ ) is said to be analytic if $\operatorname{sp}(\phi)$ (resp., $\operatorname{sp}(\lambda)$ ) is nonnegative. A measure in $M(X)$ is called quasi-invariant if its null sets are preserved under the translation by $\left\{T_{t}\right\}_{t \in R}$. Functions of unit modulus are said to be unitary functions. ${ }^{1}$

Let $\mu$ be a positive measure in $M(X)$. For each $t$ in $R$, we let $\mathfrak{M}_{t}$ be the closure in $L^{2}(\mu)$ of those functions in $C_{0}(X)$ whose spectra are contained in $(t, \infty)$. The distant future in $L^{2}(\mu)$ is defined to be the intersection $\bigwedge_{t \in R} \mathfrak{M}_{t}$.

We refer the reader to [4 and 12] for the basic facts about spectra.

Received by the editors October 15, 1984 and, in revised form, July 26, 1985.

1980 Mathematics Subject Classification. Primary 28D99, 46J15, 54H20; Secondary 43 A17.

Key words and phrases. Analytic measures, quasi-invariant measures, distant future.

${ }^{1}$ Research partially supported by a grant from the Japanese Ministry of Education. 
In [4] Forelli showed that the classic F. and M. Riesz theorems hold for analytic measures in $M(X)$. More precisely, his generalization for them states that analytic measures are quasi-invariant. A part in his argument is that if a measure $\mu$ in $M(X)$ is the total variation measure of an analytic measure, then the distant future in $L^{2}(\mu)$ is the zero subspace. On the other hand, there are many positive quasiinvariant measures for which the distant future may not vanish. As a converse to F. and M. Riesz theorems, he then raised in [5 and 6] the following

PROBLEM What makes a positive measure the total variation measure of an analytic measure?

Our objective in this note is to settle the problem:

THEOREM. Let $\mu$ be a positive measure in $M(X)$. Then the distant future in $L^{2}(\mu)$ is the zero subspace if and only if $\mu$ is the total variation measure of an analytic measure.

In the almost periodic context, Helson [9] observed that the Theorem is true under the condition that $\mu$ is absolutely continuous with respect to Haar measure (see also [15]). This result is highly important for invariant subspace theory. However many quasi-invariant measures appear which are singular, and dropping absolute continuity, we cannot make his argument work. The difficulty is partly due to the fact that Szegö's theorem may not hold for such measures.

Let us show an instance of the Theorem. Fix a unit vector $w$ in the $N$ dimensional euclidean space $R^{N}$, and let $R$ act on the $N$-dimensional torus $T^{N}$ by $T_{t} \beta(x)=\beta(x+t w)$ for each $x$ in $R^{N}$, where $\beta$ is the covering homomorphism of $R^{N}$ onto $T^{N}$ defined by $\beta\left(x_{1}, \ldots, x_{N}\right)=\left(e^{i x_{1}}, \ldots, e^{i x_{N}}\right)$. Recall that the dual group of $T^{N}$ is identified with the group $Z^{N}$ of all lattice points in $R^{N}$. This isomorphism is given by $\chi_{k}(\beta(x))=\exp (i\langle k \mid x\rangle)$ for each $k$ in $Z^{N}$, where $\langle\mid\rangle$ denotes the usual inner product on $R^{N}$. It follows from above definitions that a measure $\lambda$ in $M\left(T^{N}\right)$ is analytic if and only if the Fourier-Stieltjes coefficients of $\lambda$,

$$
\hat{\lambda}(k)=\int_{T^{N}} \bar{\chi}_{k} d \lambda,
$$

vanish for all $k$ with $\langle k \mid w\rangle<0$. Observe that $\mathfrak{M}_{t}$ is the closed subspace of $L^{2}(\mu)$ generated by all $\chi_{k}$ with $\langle k \mid w\rangle>t$, if $\mu$ is a positive measure in $M\left(T^{N}\right)$.

Thus the Theorem provides the following

COROLlaRY. Let $\mu$ be a positive measure in $M\left(T^{N}\right)$. Suppose that the distant future in $L^{2}(\mu)$ is the zero subspace. Then there is a unitary function $\phi$ on $T^{N}$ such that $(\phi d \mu)^{\wedge}(k)=0$ for all $k$ with $\langle k \mid w\rangle<0$.

We remark that this Corollary is classic if $N=1$. When the ray through $w$ meets $Z^{N}, N>1$, the Corollary follows immediately from the classic case.

In the next section, we establish the notation and present some lemmas which we shall use. In $\S 3$, we study the condition that only zero lies in the distant future, and several known results are derived from it as its corollaries. After preparing some lemmas, the Theorem is proved in $\S 4$. Then an extension of Helson's existence theorem is provided. We close with some remarks in $\S 5$.

This work was completed while the author was visiting the University of California, Berkeley, and he would like to acknowledge the hospitality of the Department 
of Mathematics. In particular, the author would like to express his sincere gratitude to Professor Henry Helson for stimulating discussions. Thanks are due to the referee as well for his valuable suggestions which improved the first version of this paper.

2. Preliminaries. We first describe how a version of Ambrose's theorem allows us to assume that our setting has a special form. Some known results are modified to suit our purposes.

Let $\nu$ be a positive measure on a space $\Omega$, and let $V$ be an invertible measurable transformation on the measure space $(\Omega, \nu)$. We call $V$ a quasi-automorphism of $(\Omega, \nu)$ if $\nu$ is quasi-invariant with respect to $V$; that is, null sets are preserved under the translation by $V$. A one-parameter group of quasi-automorphisms $\left\{V_{t}\right\}_{t \in R}$ is called a quasi-flow on $(\Omega, \nu)$ if the mapping of $(t, \omega), V_{t} \omega$, is measurable on $R \times \Omega$. Two quasi-flows $\left\{V_{t}\right\}_{t \in R}$ on $(\Omega, \nu)$ and $\left\{V_{t}^{\prime}\right\}_{t \in R}$ on $\left(\Omega^{\prime}, \nu^{\prime}\right)$ are said to be isomorphic if there are two invariant null sets $N$ of $\Omega$ and $N^{\prime}$ of $\Omega^{\prime}$, and an isomorphism $T$ of $\Omega \backslash N$ onto $\Omega^{\prime} \backslash N^{\prime}$ such that $V_{t} \omega=T^{-1} V_{t}^{\prime} T \omega$ for each $t$ in $R$ and for each $\omega$ in $\Omega \backslash N$.

Suppose that $S$ is a quasi-automorphism of a probability space $(Y, \tilde{\mu})$ and that $F(y)$ is a measurable function on $Y$ for which there are two positive constants $m$ and $M$ satisfying

$$
m \leq F(y) \leq M
$$

for $\tilde{\mu}$-a.e. $y$ in $Y$. With this data it is possible to construct a so-called quasi-flow built under a function. Define a measurable subset $\tilde{X}$ of $Y \times R$ by

$$
\tilde{X}=\{(y, s) ; 0 \leq s<F(y) \text { for } y \text { in } Y\} .
$$

Let $p(y, s)$ be a positive measurable function on $\tilde{X}$. By setting $d \mu(y, s)=p(y, s)$. $d \tilde{\mu}(y) \times d s$, we obtain a positive measure $\mu$ on $\tilde{X}$ of which the total variation $\|\mu\|$ satisfies that

$$
\|\mu\|=\int_{Y} \int_{0}^{F(y)} p(y, s) d \tilde{\mu}(y) d s .
$$

Let $\tau(y, j)$ be the function on $Y \times Z$ defined by

$$
\tau(y, j)= \begin{cases}\sum_{k=0}^{j-1} F\left(S^{k} y\right) & \text { if } j \geq 1 \\ 0 & \text { if } j=0 \\ -\tau\left(S^{j} y,-j\right) & \text { if } j \leq-1\end{cases}
$$

A quasi-flow $\left\{\tilde{T}_{t}\right\}_{t \in R}$ on $(\tilde{X}, \mu)$ is defined by

$$
\tilde{T}_{t}(y, s)=\left(S^{j} y, s+t-\tau(y, j)\right)
$$

for $\mu$-a.e. $(y, s)$ in $\tilde{X}$ if $\tau(y, j) \leq s+t<\tau(y, j+1)$. This quasi-flow is said to be built $u p$ by $(Y, \tilde{\mu}, F(y), p(y, s), S)$, and is an analogue of the one introduced by Ambrose $[\mathbf{1}]$.

Let $X, M(X)$, and $\left\{T_{t}\right\}_{t \in R}$ be as in $\S 1$, and let $\mu$ be a positive quasi-invariant measure in $M(X)$. Then, for an invariant subset $E$ of $X$, the restriction $\mu_{E}$ of $\mu$ to $E$ is also quasi-invariant. We denote by $P$ the set of all fixed points of $\left\{T_{t}\right\}_{t \in R}$. Suppose that $E$ is an invariant subset of $X \backslash P$ with $\mu(E)>0$. Then $\left\{T_{t}\right\}_{t \in R}$ may be regarded as a quasi-flow on the measure space $\left(X, \mu_{E}\right)$ without fixed points. We notice that such quasi-flow is proper, that is, if a Baire set is not null, then it has a 
Baire subset $B$ so that $\mu_{E}\left((X \backslash B) \cap T_{t_{0}} B\right)>0$ for some $t_{0}$ in $R$ (cf. [2, Definition 7]). Kubo and others have shown that there is a sequence $\left\{E_{j} ; j=1,2, \ldots\right\}$ of disjoint invariant sets such that $X \backslash P=\bigcup_{j=1}^{\infty} E_{j}$ and each quasi-flow $\left\{T_{t}\right\}_{t \in R}$ on $\left(X, \mu_{E_{j}}\right)$ is, in a measurable fashion, isomorphic to a quasi-flow built under a function (see [11, Theorem 3 and Remark 3.1] and see also [16] for minimal flows).

On the other hand, it is easy to see that if $\mu(P)>0$, then the positive measure $\mu_{P}$ is analytic and the distant future in $L^{2}\left(\mu_{P}\right)$ is the zero subspace. Therefore, by putting $\mu_{j}=\mu_{E_{j}}$, we obtain the following

LEMMA 1. Let $\mu$ be a positive quasi-invariant measure in $M(X)$, and let $P$ be the set of all fixed points of $\left\{T_{t}\right\}_{t \in R}$. Then there is a sequence $\left\{\mu_{j} ; j=1,2, \ldots\right\}$ of positive quasi-invariant measures in $M(X)$ satisfying

(i) the quasi-flow $\left\{T_{t}\right\}_{t \in R}$ on $\left(X, \mu_{j}\right)$ is isomorphic to a quasi-flow built under a function;

(ii) $\mu=\mu_{P}+\sum_{j=1}^{\infty} \mu_{j}$ and $\left\{\mu_{j}\right\}$ are mutually singular to one another; consequently, $\mu$ is the total variation measure of an analytic measure if and only if every $\mu_{j}$ is, and

(iii) the distant future in $L^{2}(\mu)$ is the zero subspace if and only if every distant future in $L^{2}\left(\mu_{j}\right)$ is.

It is convenient to write $M Q(X)$ for the class of all positive quasi-invariant measures $\mu$ in $M(X)$ for which the quasi-flow $\left\{T_{t}\right\}_{t \in R}$ on $(X, \mu)$ is isomorphic to a quasi-flow $\left\{\tilde{T}_{t}\right\}_{t \in R}$ built up by $(Y, \tilde{\mu}, F(y), p(y, s), S)$. By Lemma 1 , there is no essential loss of generality from restricting to $M Q(X)$ in what follows. For $\mu$ in $M Q(X)$, we will always identify $X,\left\{T_{t}\right\}_{t \in R}$, and $d \mu$ with $\tilde{X},\left\{\tilde{T}_{t}\right\}_{t \in R}$, and $p(y, s) d \tilde{\mu}(y) \times d s$, respectively. However, since we may not consider continuity on $\tilde{X}$, it is helpful to note a property of the distant future in $L^{2}(\mu)$. Let $\mathfrak{M}_{t}$ be as in $\S 1$, and let $\mathcal{L}_{(t, \infty)}$ be the space of all functions $\phi$ in $L^{\infty}(\mu)$ whose spectra are contained in $(t, \infty)$. Then $\mathfrak{M}_{t}$ equals the closure $\left[\mathcal{L}_{(t, \infty)}\right]_{2}$ of $\mathcal{L}_{(t, \infty)}$ in $L^{2}(\mu)$. In fact, if $\phi$ lies in $\left[\mathcal{L}_{(t, \infty)}\right]_{2} \ominus \mathfrak{M}_{t}$, then $\operatorname{sp}(\bar{\phi} \cdot d \mu)$ is contained in $[-t, \infty)$. This implies that $\phi$ is orthogonal to $\mathcal{L}_{(t, \infty)}$ in $L^{2}(\mu)$ (cf. [4, pp. 50-51]), so $\phi$ must be null. Thus we may define the distant future without the aid of continuous functions.

The measurable transformation $\sigma$ of $Y \times R$ onto itself is defined by

$$
\sigma(y, t)=(S y, t-F(y))
$$

for $d \tilde{\mu} \times d t$-a.e. $(y, t)$ in $Y \times R$. Observe that the hypotheses on $F$ imply that $Y \times R$ is the disjoint union $\bigcup_{j=-\infty}^{\infty} \sigma^{j}(X)$. Define the mapping $\pi$ of $Y \times R$ onto $X$ by

$$
\pi(y, t)=\left(S^{j} y, t-\tau(y, j)\right)
$$

if $\tau(y, j) \leq t<\tau(y, j+1)$ for $d \tilde{\mu} \times d t$-a.e. $(y, t)$ in $Y \times R$. Every function $\phi$ on $X$ has the automorphic extension to $Y \times R$ defined by

$$
\phi^{\#}(y, t)=\phi \circ \pi(y, t)
$$

for $d \tilde{\mu} \times d t$-a.e. $(y, t)$ in $Y \times R$. Similarly the measure $d \mu(y, s)=p(y, s) d \tilde{\mu}(y) \times d s$ has also the automorphic extension $\mu^{\#}$ of $\sigma$-finite measure on $Y \times R$ defined by

$$
\mu^{\#}(E)=\sum_{j=-\infty}^{\infty} \mu \circ \pi\left(E \cap \sigma^{j}(X)\right)
$$

for each measurable subset $E$ of $Y \times R$. 
LEMMA 2. Let $\mu$ be a measure in $M Q(X)$ associated with $(Y, \tilde{\mu}, F(y), p(y, s), S)$. Then there is a positive function $U(y, t)$ in $L^{1}\left(d \tilde{\mu} \times d t /\left(1+t^{2}\right)\right)$ such that the automorphic extension $\mu^{\#}$ of $\mu$ may be represented as

$$
d \mu^{\#}(y, t)=U(y, t) d \tilde{\mu}(y) \times d t .
$$

Proof. Let $\tilde{\rho}(y)$ be the Radon-Nikodym derivative $d \tilde{\mu} \circ S / d \tilde{\mu}(y)$, and define the measurable function $U(y, t)$ on $Y \times R$ by

$$
U(y, t)=p^{\#}(y, t) \times \begin{cases}\tilde{\rho}(y) \cdots \tilde{\rho}\left(S^{j-1} y\right) & \text { if } j \geq 1 \\ 1 & \text { if } j=0 \\ {\left[\tilde{\rho}\left(S^{-1} y\right) \cdots \tilde{\rho}\left(S^{j} y\right)\right]^{-1}} & \text { if } j \leq-1\end{cases}
$$

for $d \tilde{\mu} \times d t$-a.e. $(y, t)$ in $\sigma^{j}(X)$. We see easily that $d \mu^{\#}(y, t)=U(y, t) d \tilde{\mu}(y) \times d t$. Let $m$ be as in (2.1). Observe then that $|t| \geq m \cdot \min (|j|,|j-1|)$ on $\sigma^{j}(X)$. From this fact, we find a constant $C_{0}$ so that $\left(1+t^{2}\right)^{-1} \leq C_{0}\left(1+j^{2}\right)^{-1}$ on $\sigma^{j}(X)$. Since $\mu^{\#}\left(\sigma^{j}(X)\right)=\mu(X)$, we see that

$$
\begin{aligned}
\int_{Y} \int_{-\infty}^{\infty} U(y, t) d \tilde{\mu}(y) \frac{d t}{1+t^{2}} & =\iint_{Y \times R} \frac{1}{1+t^{2}} d \mu^{\#}(y, t) \\
& \leq \sum_{j=-\infty}^{\infty} \iint_{\sigma^{j}(X)} \frac{1}{1+t^{2}} d \mu^{\#}(y, t) \\
& \leq C_{0} \cdot\|\mu\| \cdot \sum_{j=-\infty}^{\infty} \frac{1}{1+j^{2}}<\infty
\end{aligned}
$$

Hence $U(y, t)$ lies in $L^{1}\left(d \tilde{\mu} \times d t /\left(1+t^{2}\right)\right)$, and this completes the proof.

We denote by $H^{p}(d t), 1 \leq p \leq \infty$, the usual Hardy spaces on $R$. Let

$$
H^{\infty}\left(d t /\left(1+t^{2}\right)\right)=H^{\infty}(d t)
$$

that is, the space of all the boundary functions of bounded analytic functions in the upper half-plane. The closure of $H^{\infty}\left(d t /\left(1+t^{2}\right)\right)$ in $L^{p}\left(d t /\left(1+t^{2}\right)\right), 1 \leq p<\infty$, is denoted by $H^{p}\left(d t /\left(1+t^{2}\right)\right)$. Recall that $f(t)$ lies in $H^{p}\left(d t /\left(1+t^{2}\right)\right)$ if and only if $f(t) \cdot(t+i)^{-2 / p}$ lies in $H^{p}(d t)$. Let $H_{i}^{p}\left(d t /\left(1+t^{2}\right)\right)$ denote the space of all functions in $H^{p}\left(d t /\left(1+t^{2}\right)\right)$ which vanish at $i$. Then it follows easily that $H^{1}(d t)$ is the space of all $g(t) /\left(1+t^{2}\right)$ for $g$ in $H_{i}^{p}\left(d t /\left(1+t^{2}\right)\right)$. This fact will be used in the forthcoming sections. Recall that the class of all continuous functions in $H^{p}(d t)$ with $|f(t)|=O\left(t^{-2}\right)$, as $|t| \rightarrow 0$, is dense in $H^{p}(d t), 1 \leq p<\infty$ (cf. [8, Chapter II, §3]).

Let $\mu$ be a measure in $M Q(X)$ associated with $(Y, \tilde{\mu}, F(y), p(y, s), S)$, and let $\mu^{\#}$ be the automorphic extension of $\mu$ to $Y \times R$. Define the mapping of $L^{1}\left(\mu^{\#}\right)$ to $L^{1}(\mu)$ by

$$
\Phi(f)(y, s)=\sum_{j=-\infty}^{\infty} f \circ \sigma^{j}(y, s)
$$

for $\mu$-a.e. $(y, s)$ in $X$. Let $y^{\infty}\left(\mu^{\#}\right)$ be the space of all functions $f$ in $L^{1}\left(\mu^{\#}\right)$ such that

$$
N_{\infty}(f)=\sum_{j=-\infty}^{\infty} \operatorname{ess.sup}\left\{\left|f \circ \sigma^{j}(y, s)\right| ;(y, s) \text { lies in } X\right\}
$$


is bounded and the function of $t, f(y, t)$, lies in $H^{1}(d t)$ for $\tilde{\mu}$-a.e. $y$ in $Y$. Then $\mathfrak{H}^{\infty}\left(\mu^{\#}\right)$ becomes a Banach space with the norm $N_{\infty}$. Recall that $\mathcal{L}_{(0, \infty)}$ denotes the space of all functions in $L^{\infty}(\mu)$ which have positive spectra. It is easy to see that the restriction of $\Phi$ to $\gamma^{\infty}\left(\mu^{\#}\right)$ is a bounded linear mapping of $\varkappa^{\infty}\left(\mu^{\#}\right)$ into $\mathcal{L}_{(0, \infty)}$ whose norm is at most one.

The next lemma follows from the definition of spectra, so the proof is omitted.

LEMMA 3. Let $\mu, \nvdash^{\infty}\left(\mu^{\#}\right)$, and $\mathcal{L}_{(0, \infty)}$ be as above, and let $U(y, t)$ be the function defined in (2.4). Then for a function $\phi$ in $L^{1}(\mu)$, the following assertions are equivalent:

(i) $\phi d \mu$ is an analytic measure in $M(X)$,

(ii) $\phi d \mu$ is orthogonal to $\mathcal{L}_{(0, \infty)}$,

(iii) $\phi^{\#} d \mu^{\#}$ is orthogonal to $\psi^{\infty}\left(\mu^{\#}\right)$, and $Y$.

(iv) the function of $t, \phi^{\#}(y, t) U(y, t)$, belongs to $H^{1}\left(d t /\left(1+t^{2}\right)\right)$ for $\tilde{\mu}$-a.e. $y$ in

We now suppose that $\mu$ is a positive quasi-invariant measure in $M(X)$. Let $H^{\infty}(\mu)$ be, for obvious reasons, the space of all analytic functions in $L^{\infty}(\mu)$. It follows from the definition of spectra that $\phi$ lies in $H^{\infty}(\mu)$ if and only if the function of $t, \phi\left(T_{t} x\right)$, lies in $H^{\infty}\left(d t /\left(1+t^{2}\right)\right)$ for $\mu$-a.e. $x$ in $X$. Then $H^{\infty}(\mu)$ is a closed subalgebra of $L^{\infty}(\mu)$ containing constants.

The following direct consequence of Lemmas 1 and 3 will be used in what follows.

LEMMA 4. Let $\mu$ be a positive quasi-invariant measure in $M(X)$, and let $H^{\infty}(\mu)$ be as above. Suppose that $\phi d \mu$ is analytic for some $\phi$ in $L^{1}(\mu)$. Then $\theta \phi d \mu$ is also analytic for each $\theta$ in $H^{\infty}(\mu)$.

We refer to [8] for results about classical Hardy spaces on $R$. Analyticity in our setting can be found in [10 and $\mathbf{7}$, Chapter VII].

3. Distant future. We characterize the circumstances under which the distant future is the zero subspace by means of the automorphic extension of a measure in $M Q(X)$. By Lemma 1, our result yields several known facts when it is specialized suitably.

PROPOSITION 1. Let $\mu$ be a measure in $M Q(X)$ associated with $(Y, \tilde{\mu}, F(y)$, $p(y, s), S)$, and let $U(y, t)$ be the function on $Y \times R$ defined in (2.4). Then the following assertions are equivalent:

(i) the distant future in $L^{2}(\mu)$ is the zero subspace,

(ii) the function of $t, \log U(y, t)$, belongs to $L^{1}\left(d t /\left(1+t^{2}\right)\right)$ for $\tilde{\mu}$-a.e. $y$ in $Y$, and

(iii) for a given $\varepsilon>0$, there is a function $\phi$ in $L^{\infty}(\mu)$ such that $\|1-|\phi|\|_{\infty}<\varepsilon$, and $\phi d \mu$ is an analytic measure. In particular, we can make such $\phi$ satisfy that $-\varepsilon<\log |\phi(x)| \leq 0$ for $\mu$-a.e. $x$ in $X$.

ProOF. (i) implies (ii). Observe first that it follows from Lemma 2 and Fubini's theorem that the function of $t, U(y, t)$, lies in $L^{1}\left(d t /\left(1+t^{2}\right)\right)$ for $\tilde{\mu}$-a.e. $y$ in $Y$. We let $E_{0}$ be the set of all $y$ in $Y$ satisfying

$$
\int_{-\infty}^{\infty} \log U(y, t) \frac{d t}{1+t^{2}}=-\infty
$$


Suppose that $\tilde{\mu}\left(E_{0}\right)$ is positive. Since

$$
U(y, t+F(y))=U(S y, t) \frac{d \tilde{\mu} \circ S}{d \tilde{\mu}}(y)
$$

and

$$
U(y, t-F(y))=U\left(S^{-1} y, t\right) \frac{d \tilde{\mu} \circ S^{-1}}{d \tilde{\mu}}(y),
$$

we see that $S y$ and $S^{-1} y$ lie in $E_{0}$ for $\tilde{\mu}$-a.e. $y$ in $E_{0}$. So $E_{0}$ is invariant with respect to $S$. If we put $E=\left\{(y, s) ; 0 \leq s<F(y)\right.$ for $y$ in $\left.E_{0}\right\}$, then $\mu(E)$ is also positive and $E$ is invariant with respect to $\left\{T_{t}\right\}_{t \in R}$. Notice that $I_{E}^{\#}(y, t)=I_{E_{0}}(y)$ for $d \tilde{\mu} \times d t$-a.e. $(y, t)$ in $Y \times R$, where $I_{E}$ and $I_{E_{0}}$ denote the characteristic functicns of $E$ and $E_{0}$, respectively.

Let us show that the distant future in $L^{2}(\mu)$ includes $I_{E} L^{2}(\mu)$, the space of all $I_{E} \phi$ for $\phi$ in $L^{2}(\mu)$. In fact, for each $r$ in $R$, let $\psi$ be a function $L^{2}(\mu)$ which is orthogonal to $\mathfrak{M}_{r}$. Notice that if $q(y)$ lies in $L^{\infty}(\tilde{\mu})$ and if $f$ lies in $H_{i}^{\infty}\left(d t /\left(1+t^{2}\right)\right)$, then the function on $X, \Phi\left(e^{i r t} I_{E_{0}}(y) q(y) f(t)\left(1+t^{2}\right)^{-1}\right)(x)$, belongs to $\mathfrak{M}_{r}$. Since

$$
\begin{aligned}
0 & =\int_{X} \bar{\psi}(x) \Phi\left(e^{i r t} I_{E_{0}}(y) q(y) f(t)\left(1+t^{2}\right)^{-1}\right)(x) d \mu(x) \\
& =\int_{Y} \int_{-\infty}^{\infty} \bar{\psi}^{\#}(y, t) I_{E}^{\#}(y, t) e^{i r t} q(y) f(t) U(y, t) d \tilde{\mu}(y) \frac{d t}{1+t^{2}} \\
& =\int_{Y} q(y)\left[\int_{-\infty}^{\infty}\left(\bar{\psi} I_{E}\right)^{\#}(y, t) e^{i r t} f(t) U(y, t) \frac{d t}{1+t^{2}}\right] d \tilde{\mu}(y)
\end{aligned}
$$

for all $q$ in $L^{\infty}(\tilde{\mu})$, we may choose a $\tilde{\mu}$-null set $N_{f}$ so that

$$
\int_{-\infty}^{\infty}\left(\bar{\psi} I_{E}\right)^{\#}(y, t) e^{i r t} f(t) U(y, t) \frac{d t}{1+t^{2}}=0
$$

for each $y$ in $Y \backslash N_{f}$. Observe that $H_{i}^{\infty}\left(d t /\left(1+t^{2}\right)\right)$ has a sequence which generates $H_{i}^{\infty}\left(d t /\left(1+t^{2}\right)\right)$ in the weak*-topology of $L^{\infty}\left(d t /\left(1+t^{2}\right)\right)$. This implies that there is a $\tilde{\mu}$-null set $N$ such that (3.2) holds for all $f$ in $H_{i}^{\infty}\left(d t /\left(1+t^{2}\right)\right)$ and all $y$ in $Y \backslash N$. On the other hand, it follows from (3.1) and Szegö's theorem that the closure of $H_{i}^{\infty}\left(d t /\left(1+t^{2}\right)\right)$ in $L^{2}\left(U(y, t) d t /\left(1+t^{2}\right)\right)$ is $L^{2}\left(U(y, t) d t /\left(1+t^{2}\right)\right)$ for $\tilde{\mu}$-a.e. $y$ in $E_{0}$, so we can see by (3.2) that $\left(\psi I_{E}\right)^{\#}(y, t)=0$ on $Y \times R$. Therefore, $\psi I_{E}$ must be null in $L^{2}(\mu)$. This shows that the nonzero subspace $I_{E} L^{2}(\mu)$ is contained in $\mathfrak{M}_{r}$ for all $r$ in $R$. Consequently, the distant future in $L^{2}(\mu)$ includes $I_{E} L^{2}(\mu)$, thus we have a contradiction.

(ii) implies (iii). It follows from (ii) and a property of outer functions that there is a function $h(y, t)$ in $L^{1}\left(d \tilde{\mu} \times d t /\left(1+t^{2}\right)\right)$ satisfying that $|h(y, t)|=U(y, t)$ and the function of $t, h(y, t)$, lies in $H^{1}\left(d t /\left(1+t^{2}\right)\right)$ for $\tilde{\mu}$-a.e. $y$ in $Y$. Let $\varepsilon>0$ be given, and define

$$
v(y, t)= \begin{cases}1 & \text { if }(y, t) \text { lies in } X, \\ c j^{-2} & \text { if }(y, t) \text { lies in } \sigma^{j}(X), j \neq 0,\end{cases}
$$

where the constant $c$ satisfies $0<c \sum_{j \neq 0} j^{-2}<\varepsilon$. Since $F(y)$ is bounded away from zero, an easy calculation shows that the function of $t, \log v(y, t)$, lies in $L^{1}\left(d t /\left(1+t^{2}\right)\right)$. By the same way as above, we may find a function $f(y, t)$ in $L^{1}(d \tilde{\mu} \times d t)$ such that $|f(y, t)|=v(y, t)$ and the function of $t, f(y, t)$, lies in $H^{1}(d t)$. 
Let us show that the bounded function, $\phi=\Phi\left(f h U^{-1}\right)$, has the desired properties. In fact, if $\psi$ lies in $\mathcal{L}_{(0, \infty)}$, then we see that

$$
\begin{aligned}
\int_{X} \psi(x) \phi(x) d \mu(x) & =\iint_{Y \times R} \psi^{\#}(y, t) f(t) h(y, t) U^{-1}(y, t) d \mu^{\#}(y, t) \\
& =\int_{Y} \int_{-\infty}^{\infty} \psi^{\#}(y, t) f(t) h(y, t) d \tilde{\mu}(y) d t \\
& =0
\end{aligned}
$$

by (2.4). Therefore by Lemma 3 we see that $\phi d \mu$ is analytic. On the other hand, since $h(y, t) U^{-1}(y, t)$ is a unitary function on $Y \times R$, it follows from (2.5) and (3.3) that $\phi$ satisfies $\|1-|\phi|\|_{\infty}<\varepsilon$. Furthermore, let $\varepsilon_{1}>0$ satisfy

$$
-\varepsilon<\log \left(1-\varepsilon_{1}\right) /\left(1+\varepsilon_{1}\right)
$$

for a given $\varepsilon>0$. By what we have just shown, there is a function $\phi_{1}$ such that $\phi_{1} d \mu$ is analytic and $\left\|1-\left|\phi_{1}\right|\right\|_{\infty}<\varepsilon_{1}$. Since $\left(1-\varepsilon_{1}\right) /\left(1+\varepsilon_{1}\right)<\left|\phi_{1}(x)\right| /\left(1+\varepsilon_{1}\right)<1$, the function $\phi=\phi_{1}(x) /\left(1+\varepsilon_{1}\right)$ satisfies the desired inequality.

(iii) implies (i). Let $\phi$ be a function such that $\frac{1}{2}<|\phi(x)|<\frac{3}{2}$ and $\phi d \mu$ is analytic. Since $\phi d \mu$ is analytic, the distant future in $L^{2}(|\phi| d \mu)$ is the zero subspace (see [4, Theorem 2]). Since the norms in $L^{2}(\mu)$ and $L^{2}(|\phi| d \mu)$ are equivalent, we see that (i) holds, and the proof is complete.

The next corollary follows from Lemma 1 and Proposition 1.

COROLLARY 1 (FORELLI [5]). Let $\mu$ be a positive quasi-invariant measure in $M(X)$. Suppose that the distant future in $L^{2}(\mu)$ is the zero subspace. Then there is a function $\phi$ such that $|\phi| \leq 1, \mu$ is absolutely continuous with respect to $\phi d \mu$, and $\phi d \mu$ is analytic.

Let $\nu$ be an invariant ergodic probability measure in $M(X)$. Then it is wellknown that $H^{\infty}(\nu)$ is a weak*-Dirichlet algebra in $L^{\infty}(\nu)$, so Szegö's theorem holds for $H^{\infty}(\nu)$ (cf. [12, Theorem I]). This implies that if $\log u$ lies in $L^{1}(\nu)$ for a nonnegative function $u$ in $L^{2}(\nu)$, then there is a function $\phi$ in $H^{2}(\nu)$ so that $|\phi|=u$, where $H^{2}(\nu)$ is defined to be the closure of $H^{\infty}(\nu)$ in $L^{2}(\nu)$.

Futhermore, we have

COROLLARY 2 (HELSON [9]). Let $w$ be a nonnegative function in $L^{2}(\nu)$ satisfying

$$
\int_{-\infty}^{\infty} \log w\left(T_{t} x\right) \frac{d t}{1+t^{2}}>-\infty
$$

for $\nu$-a.e. $x$ in $X$. Then there is a function $\phi$ in $H^{2}(\nu)$ such that $|\phi|=w$.

Proof. Recall that $\nu$ belongs to $M Q(X)$ and the ergodic flow $\left\{T_{t}\right\}_{t \in R}$ on $(X, \nu)$ is represented by a flow built up by $(Y, \tilde{\nu}, F(x), 1, S)$ for some invariant ergodic measure $\tilde{\nu}$ on $Y$ (see [1, Theorem 2]). If we put $d \mu(x)=w(x) d \nu(x)$, then $d \mu^{\#}(y, t)=w^{\#}(y, t) d \tilde{\nu}(y) \times d t$ by (2.4). So it follows from (3.4) and Proposition 1 that there is a function $\psi$ in $L^{\infty}(\nu)$ such that $\psi$ is bounded away from zero and $\psi w d \nu$ is analytic. Since $\log |\psi|^{-1}$ is also bounded, we may choose a function $\theta$ in $H^{\infty}(\nu)$ such that $|\theta|=|\psi|^{-1}$ by Szegö's theorem. Therefore it follows from Lemma 4 that the function $\phi=\theta \psi w$ satisfies the desired property. 
We notice that if $\log w$ lies in $L^{1}(\nu)$, then (3.4) holds by Fubini's theorem. However an example shows that the converse implication is false $[\mathbf{1 0}$, Chapter 3 , $\S 3]$. We will extend Corollary 2 to the case of arbitrary positive quasi-invariant measures in the next section.

Let $\mu$ be a positive quasi-invariant measure in $M(X)$. We write $\rho(x, t)$ for the Radon-Nikodym derivative $d \mu \circ T_{t} / d \mu(x)$. It was shown that $\rho(x, t)$ may be chosen to be measurable with respect to $d \mu \times d t$ (see $[13, \S 2])$. Suppose that $\mu$ is a measure in $M Q(X)$ associated with $(Y, \tilde{\mu}, F(y), p(y, s), S)$. It follows from (2.3) and (2.4) that if $E$ is a Baire subset of $X$, then we have

$$
\begin{aligned}
\mu \circ T_{t}(E) & =\iint_{Y \times R} I_{E}(y, s-t) d \mu^{\#}(y, s) \\
& =\iint_{Y \times R} I_{E}(y, s-t) U(y, s) d \tilde{\mu}(y) d s \\
& =\iint_{X} I_{E}(y, s) U(y, s+t) U^{-1}(y, s) d \mu(y, s)
\end{aligned}
$$

for each $t$ in $R$ by regarding $E$ as a subset of $Y \times R$. This shows that

$$
\rho(x, t)=\rho((y, s), t)=U(y, s-t) U^{-1}(y, s) \text { for } \mu \text {-a.e. } x=(y, s) \text { in } X \text {. }
$$

Therefore the function of $t, \log \rho(x, t)$, lies in $L^{1}\left(d t /\left(1+t^{2}\right)\right)$ for $\mu$-a.e. $x$ in $X$ if and only if assertion (ii) of Proposition 1 holds. Thus the next corollary follows from Lemma 1.

COROLLARY 3 (MUHLY [13]). Let $\mu$ be a positive quasi-invariant measure in $M(X)$, and let $\rho(x, t)$ be defined as above. Then the distant future in $L^{2}(\mu)$ is the zero subspace if and only if the function of $t, \log \rho(x, t)$, lies in $L^{1}\left(d t /\left(1+t^{2}\right)\right)$ for $\mu$-a.e. $x$ in $X$.

4. Total variation measures of analytic measures. Let $\mu$ be a positive measure in $M(X)$ for which the distant future in $L^{2}(\mu)$ vanishes. Then we have seen by Proposition 1 that there is a function $\phi$ such that $\phi d \mu$ is analytic and $|\phi|$ is close to 1 as near as desired. However, what we want to know is whether such $\phi$ may be always chosen with $|\phi|=1$. There is a tedious way to fill this gap.

We begin with

LEMMA 5. Let $\mu$ be a measure in $M Q(X)$ associated with $(Y, \tilde{\mu}, F(y), p(y, t), S)$, and let $u$ be $a$ bounded real-valued function on $X$. Suppose that $u$ satisfies

$$
\int_{0}^{F(y)} u(y, s) d s=0
$$

for $\tilde{\mu}$-a.e. $y$ in $Y$. Then there is a function $\phi$ in $H^{\infty}(\mu)$ such that $|\phi|=e^{u}$.

Proof. Define the bounded function $h(y, t)$ on $Y \times R$ by

$$
h(y, t)= \begin{cases}u(y, t) & \text { if }(y, t) \text { lies in } X \\ 0 & \text { otherwise. }\end{cases}
$$

Let us consider the Hilbert transform $\tilde{h}(y, t)$ of $h(y, t)$; that is,

$$
\tilde{h}(y, t)=\lim _{\varepsilon \rightarrow 0} \frac{1}{\pi} \int_{|t-s|>\varepsilon} h(y, s) \frac{d s}{t-s} \text {. }
$$


The existence of this limit is assured by Fubini's theorem. Let $m$ and $M$ be as in (2.1), and let $\tau(y, t)$ be the function defined by (2.2). It follows from Plancherel's theorem (cf. [8, Chapter III, (1.9) and (1.10)]) that if $|j| \leq 1$, then

$$
\begin{aligned}
\int_{\tau(y, j)}^{\tau(y, j+1)}|\tilde{h}(y, t)| d t & \leq M\left[\int_{\tau(y, j)}^{\tau(y, j+1)}|\tilde{h}(y, t)|^{2} d t\right]^{1 / 2} \leq M\left[\int_{-\infty}^{\infty}|\tilde{h}(y, t)|^{2} d t\right]^{1 / 2} \\
& =M\left[\int_{-\infty}^{\infty}|h(y, t)|^{2} d t\right]^{1 / 2} \leq M^{2}\|u\|_{\infty}
\end{aligned}
$$

for $\tilde{\mu}$-a.e. $y$ in $Y$. This implies that

$$
\int_{Y} \int_{\tau(y, t)}^{\tau(y, j+1)}|\tilde{h}(y, t)| d \tilde{\mu} \circ S^{j+k}(y) d t \leq M^{2}\|u\|_{\infty}\|\tilde{\mu}\|
$$

for all integers $k$. We next show that

$$
\sum_{|j| \geq 2} \operatorname{ess} \cdot \sup \left\{|\tilde{h}(y, t)| ;(y, t) \text { lies in } \sigma^{j}(X)\right\}
$$

is bounded. In fact, if $(y, t)$ lies in $\sigma^{-j}(X)$ with $|j| \geq 2$, then $\tau(y, j)-F(y) \leq$ $t-s<\tau(y, j+1)$ when $0 \leq s<F(y)$. Notice that

$$
\begin{aligned}
\tilde{h}(y, t) & =\frac{1}{\pi} \int_{0}^{F(y)} h(y, s) \frac{d s}{t-s} \\
& =\frac{1}{\pi} \int_{0}^{F(y)}\left[u^{+}(y, s)-u^{-}(y, s)\right] \frac{d s}{t-s},
\end{aligned}
$$

where $u^{+}=\max (u, 0)$ and $u^{-}=\max (-u, 0)$. Since

$$
\int_{0}^{F(y)} u^{+}(y, s) d s=\int_{0}^{F(y)} u^{-}(y, s) d s
$$

for $\tilde{\mu}$-a.e. $y$ in $Y$, we see easily that

$$
\begin{aligned}
|\tilde{h}(y, t)| & \leq \frac{1}{\pi} \int_{0}^{F(y)} u^{+}(y, s)\left|\frac{1}{\tau(y, j)-F(y)}-\frac{1}{\tau(y, j+1)}\right| d s \\
& \leq \frac{1}{\pi} \int_{0}^{F(y)} u^{+}(y, s) \frac{2 M}{m^{2}(j+1)(j-1)} d s \leq c\|u\|_{\infty} \frac{1}{j^{2}-1}
\end{aligned}
$$

on $\sigma^{-j}(X)$, where $c$ is a constant independent of $j$. This shows that (4.3) holds. It follows from (4.2) and (4.3) that the function on $X$,

$$
\begin{aligned}
\Phi(h+i \tilde{h})(y, s) & =\sum_{j=-\infty}^{\infty}(h+i \tilde{h}) \circ \sigma^{j}(y, s) \\
& =\sum_{j=-\infty}^{\infty}(h+i \tilde{h})\left(S^{j} y, s-\tau(y, j)\right),
\end{aligned}
$$

belongs to $L^{1}\left(d \tilde{\mu} \circ S^{k} \times d t, X\right)$ for all integers $k$. Moreover, we see that there is a constant $C_{1}$ independent of $k$ such that

$$
\sum_{j=-\infty}^{\infty} \int_{Y} \int_{0}^{F(y)}\left|(h+i \tilde{h})\left(S^{j} y, s-\tau(y, j)\right)\right| d \tilde{\mu} \circ S^{k}(y) d s \leq C_{1} .
$$


On the other hand, by (2.5), it is easy to see that

$$
\Phi(h+i \tilde{h})^{\#}(y, t)=\sum_{j=-\infty}^{\infty}(h+i \tilde{h})\left(S^{j} y, t-\tau(y, j)\right)
$$

for $d \tilde{\mu} \times d t$-a.e. $(y, t)$ in $Y \times R$. Let $C_{0}$ be as in the proof of Lemma 2. Then we have $\left(1+t^{2}\right)^{-1} \leq C_{0}\left(1+k^{2}\right)^{-1}$ on $\sigma^{k}(X)$ for all integers $k$. Since $\tau(y, j+k)=$ $\tau(y, k)+\tau\left(S^{k} y, j\right)$, it can be seen that

$$
\begin{aligned}
& \sum_{j=-\infty}^{\infty} \int_{Y} \int_{-\infty}^{\infty}\left|(h+i \tilde{h})\left(S^{j} y, t-\tau(y, j)\right)\right| d \tilde{\mu}(y) \frac{d t}{1+t^{2}} \\
& \quad \leq C_{0} \sum_{j=-\infty}^{\infty} \sum_{k=-\infty}^{\infty} \int_{Y} \int_{\tau(y, k)}^{\tau(y, k+1)}\left|(h+i \tilde{h})\left(S^{j} y, t-\tau(y, j)\right)\right| d \tilde{\mu}(y) \frac{d t}{1+k^{2}} \\
& =C_{0} \sum_{k=-\infty}^{\infty} \frac{1}{1+k^{2}}\left[\sum_{j=-\infty}^{\infty} \int_{Y} \int_{\tau(y, k)}^{\tau(y, k+1)}\right. \\
& =C_{0} \sum_{k=-\infty}^{\infty} \frac{1}{1+k^{2}}\left[\sum_{j=-\infty}^{\infty} \int_{Y} \int_{0}^{F\left(S^{k} y\right)}\left|(h+i \tilde{h})\left(S^{j+k} y, t-\tau\left(S^{k} y, j\right)\right)\right| d \tilde{\mu}(y) d t\right] \\
& =C_{0} \sum_{k=-\infty}^{\infty} \frac{1}{1+k^{2}}\left[\sum_{j=-\infty}^{\infty} \int_{Y} \int_{0}^{F(y)}\left|(h+i \tilde{h})\left(S^{j} y, t-\tau(y, j)\right)\right| d \tilde{\mu} \circ S^{-k}(y) d t\right] \\
& \leq C_{0} C_{1} \sum_{k=-\infty}^{\infty} \frac{1}{1+k^{2}}<\infty .
\end{aligned}
$$

Then by Fubini's theorem, it is clear that $\Phi(h+i \tilde{h})^{\#}(y, t)$ belongs to $L^{1}\left(d \tilde{\mu} \times d t /\left(1+t^{2}\right)\right)$ as well as the function of $t, \Phi(h+i \tilde{h})(y, t)$, and lies in $H^{1}\left(d t /\left(1+t^{2}\right)\right)$ for $\tilde{\mu}$-a.e. $y$ in $Y$. We then set $\phi=\exp \Phi(h+i \tilde{h})$. Since $\operatorname{Re} \Phi(h+i \tilde{h})$ $=u$, the function $\phi$ in $H^{\infty}(\mu)$ satisfies $|\phi|=e^{u}$. This completes the proof.

Let $\mu$ be as in Lemma 5 , and let $m$ be the constant in (2.1). For a given $a>0$, $z(y, j)$ denotes the function on $Y \times Z$ defined by

$$
z(y, j)=\left\{\tau(y, j)+\frac{1}{2} m\right\}+i a .
$$

Since $|j| m \leq|\tau(y, j)|$, we see that $\sum_{j=-\infty}^{\infty} a\left(1+|z(y, j)|^{2}\right)^{-1}$ is bounded. This condition implies that there is a unitary function $B(y, t)$ on $Y \times R$ such that the function of $t, B(y, t)$, is a Blaschke product with zeros $\{z(y, j) ; j=0, \pm 1, \ldots\}$ for $\tilde{\mu}$-a.e. $y$ in $Y$ (cf. [8, Chapter II, (2.3)]). We denote by $P_{a}(y)$ the Poisson kernel for the point $i a$; that is,

$$
P_{a}(t)=\frac{1}{\pi} \frac{a}{a^{2}+t^{2}}
$$

To control the average of height on each segment $[0, F(y))$, we need 
LEMMA 6. Let $\{z(y, j)\}$ and $P_{a}(t)$ be as above. Then for each $\varepsilon>0$, there is a function $\psi$ in $H^{\infty}(\mu)$ satisfying

(i) $-\varepsilon<\log |\psi(x)| \leq 0$ for $\mu$-a.e. $x$ in $X$, and

(ii) if $q$ lies in $L^{\infty}(\tilde{\mu})$, then the function on $X, \psi(x) \Phi\left(q(y) P_{a}\left(t-\frac{1}{2} m\right)\right)(x)$, lies in $H^{\infty}(\mu)$.

Proof. Let $B(y, t)$ be the unitary function on $Y \times R$ defined above. For a given $\varepsilon>0$, we fix an $\varepsilon_{1}>0$ satisfying $-\varepsilon<\log \left(1-\varepsilon_{1}\right) /\left(1+\varepsilon_{1}\right)$. If $v_{1}(y, t)$ is defined by (3.3) with $\varepsilon$ replaced by $\varepsilon_{1}$, then there is a function $f(y, t)$ in $\mathcal{H}^{\infty}\left(\mu^{\#}\right)$ so that $v_{1}(y, t)=|f(y, t)|$ on $Y \times R$. Define $g(y, t)=B(y, t) f(y, t)$. Then by the same way as in the proof of Proposition 1, the function in $H^{\infty}(\mu), \psi(x)=\left(1+\varepsilon_{1}\right)^{-1} \Phi(g)(x)$, satisfies inequality (i).

On the other hand, observe that the function of $z, \psi^{\#}(y, z)$, vanishes at $\frac{1}{2} m+i a$ for $\tilde{\mu}$-a.e. $y$ in $Y$. Since the poles of $q(y) P_{a}\left(z-\frac{1}{2} m\right)$ are cancelled by corresponding zeros of $\psi^{\#}(y, z), \psi^{\#}(y, t) q(y) P_{a}\left(t-\frac{1}{2} m\right)$ belongs to $\nvdash^{\infty}\left(\mu^{\#}\right)$. Since

$$
\Phi\left(\psi^{\#}(y, t) q(y) P_{a}\left(t-\frac{1}{2} m\right)\right)(x)=\psi(x) \Phi\left(q(y) P_{a}\left(t-\frac{1}{2} m\right)\right)(x)
$$

by (2.5), we see that (ii) holds.

A property of the Poisson kernel enables us to choose a small $a>0$ such that

$$
\frac{1}{10} \int_{0}^{F(y)} P_{a}\left(t-\frac{1}{2} m\right) d t \geq \int_{0}^{F(y)} \sum_{j \neq 0} P_{a}\left(t-\frac{1}{2} m-\tau(y, j)\right) d t
$$

for $\tilde{\mu}$-a.e. $y$ in $Y$. Since $|j| m \leq|\tau(y, j)|$, we may see that the series of functions of $t, \sum_{j \neq 0} P_{a}\left(t-\frac{1}{2} m-\tau(y, j)\right)$, converges uniformly on $[0, F(y))$. We fix such an $a>0$. On the other hand, since $t^{-1} \log (1+t) \rightarrow 1$ (as $\left.t \rightarrow 0\right)$, there is a constant $b$ with $0<b \leq \frac{1}{10}$ such that if $0 \leq t<b$, then

$$
\left\{\begin{array}{l}
t \geq \log (1+t) \geq \frac{10}{11} t \\
-t \geq \log (1-t) \geq-\frac{11}{10} t .
\end{array}\right.
$$

LEMMA 7. Let $g(t)$ be a real-valued function on $[0, F(y))$ with $\|g\|_{\infty} \leq \frac{1}{10}$. Then we may choose a constant $k_{0}>0$ independent of $y$ so that if $0 \leq k \leq k_{0}$, then

$$
\begin{aligned}
& \int_{0}^{F(y)} \log \left(1+g(t)+\frac{k}{5} P_{a}\left(t-\frac{1}{2} m\right)\right) d t \\
& \quad \geq \int_{0}^{F(y)} \log \left(1+g(t)+\sum_{j \neq 0} k P_{a}\left(t-\frac{1}{2} m-\tau(y, j)\right)\right) d t
\end{aligned}
$$

and

$$
\begin{aligned}
\int_{0}^{F(y)} & \log \left(1+g(t)-\frac{k}{5} P_{a}\left(t-\frac{1}{2} m\right)\right) d t \\
\leq & \int_{0}^{F(y)} \log \left(1+g(t)-\sum_{j \neq 0} k P_{a}\left(t-\frac{1}{2} m-\tau(y, j)\right)\right) d t
\end{aligned}
$$

for $\tilde{\mu}$-a.e. $y$ in $Y$. 
Proof. We define

$$
f_{1}(t)=\frac{1}{5} P_{a}\left(t-\frac{1}{2} m\right), \quad f_{2}(t)=\sum_{j \neq 0} P_{a}\left(t-\frac{1}{2} m-\tau(y, j)\right)
$$

on $[0, F(y))$. Then by $(4.4)$, we see that $\int_{0}^{F(y)} f_{1}(t) d t \geq 2 \int_{0}^{F(y)} f_{2}(t) d t$. It follows from the assumption of $g$ that

$$
\int_{0}^{F(y)} \frac{f_{1}(t)}{1+g(t)} d t \geq 2 \frac{10}{11} \frac{9}{10} \int_{0}^{F(y)} \frac{f_{2}(t)}{1+g(t)} d t .
$$

We then choose $k_{0}>0$ such that $k_{0} \frac{10}{9} \max \left(\left\|f_{1}\right\|_{\infty},\left\|f_{2}\right\|_{\infty}\right)<b$, where $b$ is determined by (4.5). For each $k$ with $0<k<k_{0}$, we have that

$$
\begin{aligned}
\int_{0}^{F(y)} & \log \left(1+g(y)+k f_{1}(t)\right) d t-\int_{0}^{F(y)} \log (1+g(t)) d t \\
= & \int_{0}^{F(y)} \log \left(1+\frac{k f_{1}(t)}{1+g(t)}\right) d t \geq \frac{10}{11} \int_{0}^{F(y)} \frac{k f_{1}(t)}{1+g(t)} d t \\
& \geq 2\left(\frac{10}{11}\right)^{2} \frac{9}{10} \int_{0}^{F(y)} \frac{k f_{2}(t)}{1+g(t)} d t \geq \int_{0}^{F(y)} \log \left(1+\frac{k f_{2}(t)}{1+g(t)}\right) d t \\
= & \int_{0}^{F(y)} \log \left(1+g(t)+k f_{2}(t)\right) d t-\int_{0}^{F(y)} \log (1+g(t)) d t
\end{aligned}
$$

by (4.5) and (4.6). This shows one inequality, and the proof of the other is analogous.

Now we may offer a proof of our main result stated in $\S 1$.

ProOF OF THE THEOREM. Let $\mu$ be a positive measure in $M(X)$. Recall that if $\mu$ is the total variation measure of an analytic measure, then the distant future in $L^{2}(\mu)$ vanishes (cf. [4, pp. 49-50]). Then it is enough to show that the converse holds. Also recall if the distant future in $L^{2}(\mu)$ is the zero subspace, $\mu$ is quasi-invariant (see [4, Theorem 2]). Therefore Lemma 1 allows us to assume that $\mu$ is a measure in $M Q(X)$ associated with $(Y, \tilde{\mu}, F(y), p(y, s), S)$.

We choose $b_{0}>0$ such that $b_{0} \sum_{j=0}^{\infty} 5^{-j}<b$ where $b$ is determined by (4.5). Notice that $0<b \leq \frac{1}{10}$. Let $k_{0}$ be as in Lemma 7, and fix $k$ with $0<k<$ $k_{0}$ satisfying $\Phi\left(k P_{a}\left(t-\frac{1}{2} m\right)\right)(x)<b_{0}$ for $\mu$-a.e. $x$ in $X$. We then set $Q(t)=$ $k P_{a}\left(t-\frac{1}{2} m\right)$ with this $k$, and choose $\delta>0$ such that

$$
\int_{0}^{F(y)} \log (1+Q(t)) d t \geq \delta
$$

for $\tilde{\mu}$-a.e. $y$ in $Y$. It follows from (iii) of Proposition 1 that there is a function $\phi_{0}$ in $L^{\infty}(\mu)$ such that $\phi_{0} d \mu$ is analytic and

$$
-\frac{1}{2} \delta \leq \int_{0}^{F(y)} \log \left|\phi_{0}(y, t)\right| d t \leq 0
$$

for $\tilde{\mu}$-a.e. $y$ in $Y$, since $F(y)$ is bounded. We also see that a function $\psi_{0}$ in $H^{\infty}(\mu)$ satisfies property (ii) of Lemma 6 and

$$
-\frac{1}{2} \delta \leq \int_{0}^{F(y)} \log \left|\psi_{0}(y, t)\right| d t \leq 0
$$


for $\tilde{\mu}$-a.e. $y$ in $Y$. Define the measurable function $G(y)$ on $Y$ by

$$
G(y)=-\int_{0}^{F(y)} \log \left|\psi_{0} \phi_{0}(y, t)\right| d t
$$

Then $0 \leq G(y) \leq \delta$. By (4.7), the monotone convergence theorem assures that there is a function $q_{0}$ in $L^{\infty}(\tilde{\mu})$ with $0 \leq q_{0} \leq 1$ such that

$$
G(y)=\int_{0}^{F(y)} \log \left(1+q_{0}(y) Q(t)\right) d t .
$$

Observe that

$$
G(y) \leq \int_{0}^{F(y)} \log \left(1+\Phi\left(q_{0} Q\right)(y, t)\right) d t
$$

and $\left\|\Phi\left(q_{0} Q\right)\right\|_{\infty}<b_{0}$. Since by Lemma 7 ,

$$
\begin{aligned}
G(y) & =\int_{0}^{F(y)} \log \left(1+\Phi\left(q_{0} Q\right)(y, t)-\sum_{j \neq 0} q_{0}\left(S^{j} y\right) Q(t-\tau(y, j))\right) d t \\
& \geq \int_{0}^{F(y)} \log \left(1+\Phi\left(q_{0} Q\right)(y, t)-\sum_{j \neq 0} Q(t-\tau(y, j))\right) d t \\
& \geq \int_{0}^{F(y)} \log \left(1+\Phi\left(q_{0} Q\right)(y, t)-\frac{1}{5} Q(t)\right) d t .
\end{aligned}
$$

We can also find a function $q_{1}$ in $L^{\infty}(\tilde{\mu})$ with $0 \leq q_{1} \leq \frac{1}{5}$ so that

$$
G(y)=\int_{0}^{F(y)} \log \left(1+\Phi\left(q_{0} Q\right)(y, t)-q_{1}(y) Q(t)\right) d t .
$$

By induction, there is a sequence $\left\{q_{j} ; j=0,1, \ldots\right\}$ in $L^{\infty}(\tilde{\mu})$ such that $0 \leq q_{j} \leq 5^{-j}$ and

$$
G(y)=\int_{0}^{F(y)} \log \left(1+\sum_{j=0}^{n-1}(-1)^{j} \Phi\left(q_{j} Q\right)(y, t)+(-1)^{n} q_{n}(y) Q(t)\right) d t
$$

Observe that

$$
\sum_{j=0}^{\infty}\left\|(-1)^{j} \Phi\left(q_{j} Q\right)\right\|_{\infty} \leq b_{0} \sum_{j=0}^{\infty} 5^{-j}<\frac{1}{10} .
$$

Then it follows from the bounded convergence theorem that

$$
G(y)=\int_{0}^{F(y)} \log \left(1+\sum_{j=0}^{\infty}(-1)^{j} \Phi\left(q_{j} Q\right)(y, t)\right) d t .
$$

Since $\Phi$ is a bounded linear mapping of $L^{1}\left(\mu^{\#}\right)$ into $L^{1}(\mu)$, we see also that

$$
\sum_{j=0}^{\infty}(-1)^{j} \Phi\left(q_{j} Q\right)(y, t)=\Phi\left(\sum_{j=0}^{\infty}(-1)^{j} q_{j} Q\right)(y, t)
$$


for $\mu$-a.e. $(y, t)$ in $X$. Let $\theta_{0}$ be the positive bounded function defined by

$$
\theta_{0}(y, t)=1+\Phi\left(\sum_{j=0}^{\infty}(-1)^{j} q_{j} Q\right)(y, t)
$$

Then by Lemma 6 we see that $\theta_{0} \psi_{0}$ belongs to $H^{\infty}(\mu)$. It follows from Lemma 4 that $\theta_{0} \psi_{0} \phi_{0} d \mu$ is an analytic measure in $M(X)$. On the other hand, we have that

$$
\begin{aligned}
\int_{0}^{F(y)} \log \left|\theta_{0} \psi_{0} \phi_{0}(y, t)\right| d t & =\int_{0}^{F(y)} \log \theta_{0}(y, t) d t+\int_{0}^{F(y)} \log \left|\psi_{0} \phi_{0}(y, t)\right| d t \\
& =G(y)-G(y)=0
\end{aligned}
$$

for $\tilde{\mu}$-a.e. $y$ in $Y$. Since $\log \left|\theta_{0} \psi_{0} \phi_{0}(y, t)\right|$ is bounded, Lemma 5 implies that there exists a function $\phi_{1}$ in $H^{\infty}(\mu)$ such that $\left|\phi_{1}\right|=\exp \left(-\log \left|\theta_{0} \psi_{0} \phi_{0}\right|\right)$. Thus we have an analytic measure $\phi_{1} \theta_{0} \psi_{0} \phi_{0} d \mu$ which has the total variation measure $\mu$, and this completes the proof.

A method similar to that used above enables us to show another extension of Corollary 2 to Proposition 1 to arbitrary quasi-invariant measures.

Proposition 2. Let $\mu$ be a positive quasi-invariant measure in $M(X)$, and let $w$ be a nonnegative function in $L^{\infty}(\mu)$ such that

$$
\int_{-\infty}^{\infty} \log w\left(T_{t} x\right) \frac{d t}{1+t^{2}}>-\infty
$$

for $\mu$-a.e. $x$ in $X$. Then there is a function $\phi$ in $H^{\infty}(\mu)$ such that $|\phi|=w$.

ProOF. By virtue of Lemma 1 , we may assume that $\mu$ is a measure in $M Q(X)$ associated with $(Y, \tilde{\mu}, F(y), p(y, s), S)$. Our hypotheses assure that there is a function $h(y, t)$ in $L^{\infty}(d \tilde{\mu} \times d t)$ such that $|h(y, t)|=w^{\#}(y, t)$ and that the function of $t, h(y, t)$, lies in $H^{\infty}\left(d t /\left(1+t^{2}\right)\right)$ for $\tilde{\mu}$-a.e. $y$ in $Y$. Let $\delta$ be as in (4.7). By using the auxiliary function defined in (3.3), we may find a function $\phi_{2}$ in $L^{\infty}(\mu)$ so that $\phi_{2} w$ lies in $H^{\infty}(\mu)$ and that $-\frac{1}{2} \delta \leq \log \left|\phi_{2}(x)\right| \leq 0$ on $X$. Let $\psi_{0}$ be the same function in the proof of the Theorem. Then we similarly choose a positive function $\theta_{1}$ such that $\theta_{1} \psi_{0}$ lies in $H^{\infty}(\mu)$ and

$$
\int_{0}^{F(y)} \log \left|\theta_{1} \psi_{0} \phi_{2}(y, t)\right| d t=0
$$

for $\tilde{\mu}$-a.e. $y$ in $Y$. Therefore it follows from Lemma 5 that there is a function $\phi_{3}$ in $H^{\infty}(\mu)$ so that $\left|\phi_{3}\right|=\left|\theta_{1} \psi_{0} \phi_{2}\right|^{-1}$. Hence $\phi_{3} \theta_{1} \psi_{0} \phi_{2} w$ is a desired analytic function on $X$, and the proof is complete.

5. Remarks. (a) Let $A$ be the closed subalgebra of all analytic functions in $C_{0}(X)$, and let $A_{0}$ be the subspace of all functions in $A$ whose spectra are positive. Then $A_{0}$ is an ideal of $A$. Recall that the space of all analytic measures coincides with the annihilator in $M(X)$ of $A_{0}$ (see [4, Proposition 2]). Since our proofs do not mention the continuity of $\left\{T_{t}\right\}_{t \in R}$, we may extend the Theorem to more general quasi-flows. However, for the above reason, analytic measures have an important significance in relation to $A$. So it seems that our restriction is proper.

(b) Let $\mu$ be a positive measure in $M(X)$ for which the distant future in $L^{2}(\mu)$ is the zero subspace. We do not know what kind of analytic measures have the 
total variation measure $\mu$. For instance, suppose that $\mu$ is a measure in $M Q(X)$ associated with $(Y, \tilde{\mu}, F(y), p(y, s), S)$. The Theorem asserts that there is a unitary function $\theta$ on $X$ such that the function of $t, \theta^{\#}(y, t) U(y, t)$, lies in $H^{1}\left(d t /\left(1+t^{2}\right)\right)$ for $\tilde{\mu}$-a.e. $y$ in $Y$, where $U(y, t)$ is the function in (2.4). When can we choose such $\theta$ with the property that the function of $t, \theta^{\#}(y, t) U(y, t)$, is an outer function in $H^{1}\left(d t /\left(1+t^{2}\right)\right)$ ? This question is connected with a longstanding problem, and perhaps analytic cocycles would play an important role in this direction (cf. [10, Chapter 5, §4 and 7, Chapter VII, Theorem 7.8]).

\section{REFERENCES}

1. W. Ambrose, Representation of ergodic flows, Ann. of Math. (2) 42 (1941), 723-739.

2. W. Ambrose and S. Kakutani, Structure and continuity of measurable flows, Duke Math. J. 9 (1942), 25-42.

3. K. deLeeuw and I. Glicksberg, Quasi-invariance and analyticity of measures on compact groups, Acta Math. 109 (1963), 179-205.

4. F. Forelli, Analytic and quasi-invariant measures, Acta Math. 118 (1967), 33-59.

5. What makes a positive measure the total variation measure of an analytic measure?, J. London Math. Soc. 2 (1970), 713-718.

6. __ Fourier theory and flows, Actes Congrés Internat. Math. 2 (1970), 456-457.

7. T. Gamelin, Uniform algebras, Prentice-Hall, Englewood Cliffs, N.J., 1969.

8. J. Garnett, Bounded analytic functions, Academic Press, New York, 1981.

9. H. Helson, Compact groups with ordered duals. IV, Bull. London Math. Soc. 5 (1973), 67-69.

10. , Analyticity on compact abelian groups, Algebras in Analysis, Academic Press, London, 1975, pp. 1-62.

11. I. Kubo, Quasi-flows, Nagoya Math. J. 35 (1969), 1-30.

12. P. Muhly, Function algebras and flows, Acta Sci. Math. (Szeged) 35 (1973), 111-121.

13. _ The distant future, Indiana Math. J. 24 (1974), 149-159.

14. _ Ergodic Hardy spaces and duality, Michigan Math. J. 25 (1978), 317-323.

15. J.-I. Tanaka, A certain class of total variation measures of analytic measures, Pacific J. Math. 82 (1979), 547-558.

16. __ Quasi-invariant measures and maximal algebras on minimal flows, Michigan Math. J. 29 (1982), 199-211.

Department of MAThematics, UNiversity of CALIFornia, Berkeley, CaliforNIA 94720

Department of Mathematics, TSURU University, TSURU City, Yamanashi-Ken 402, JAPAN 